\title{
A new species of Longicoelotes (Araneae, Agelenidae) from China, with the first description of the male of L. kulianganus (Chamberlin, 1924)
}

\author{
Xiaoqing Zhang ${ }^{1,2}$, Zhe Zhao' \\ I Institute of Zoology, Chinese Academy of Sciences, Beijing 100101, China 2 Southeast Asia Biodiversity \\ Research Institute, Chinese Academy of Sciences, Yezin, Nay Pyi Taw 05282, Myanmar \\ Corresponding author: Zhe Zhao (zhaozhe@ioz.ac.cn) \\ Academic editor: Y. Marusik | Received 5 January 2017 | Accepted 10 July 2017 | Published 25 July 2017 \\ http://zoobank.org/D9169502-8C3D-443E-B51C-20E6C9A36C66 \\ Citation: Zhang X, Zhao Z (2017) A new species of Longicoelotes (Araneae, Agelenidae) from China, with the first \\ description of the male of L. kulianganus (Chamberlin, 1924). ZooKeys 686: 137-147. https://doi.org/10.3897/ \\ zookeys.686.11711
}

\begin{abstract}
A new Longicoeletes species is described from Jiangxi Province, China: L. geei sp. n. (j̊ + ). In addition, the male of L. kulianganus (Chamberlin, 1924) is described for the first time. DNA barcodes of the two species are documented for future use and as proof of molecular differences between these species.
\end{abstract}

\section{Keywords}

East Asia, description, Coelotinae, taxonomy

\section{Introduction}

The Longicoelotes was described by Wang (2002), with L. karschi Wang, 2002 from China as the type species. Wang (2003) transferred Coelotes kulianganus Chamberlin, 1924 from China and C. senkakuensis Shimojana, 2000 from Ryukyu Islands to Longicoelotes. Three species of Longicoelotes were known before the current study (World Spider Cata- 
$\log 2017)$, two of which are restricted to China. This paper provides the description of a new species based on newly collected material from Jiangxi Province, China. It also redescribes $L$. kulianganus based on specimens collected from the type locality.

\section{Material and methods}

Specimens were examined and measured with a Leica M205C stereomicroscope. Images were captured with an Olympus C7070 wide zoom digital camera mounted on an Olympus SZX12 dissecting microscope. Epigynes and male palps were examined after dissection. Epigynes were cleared by boiling in $10 \% \mathrm{KOH}$ solution before taking photos. All measurements are given in millimeters. Leg measurements are given as: total length (femur, patella + tibia, metatarsus, tarsus). Only structures (palp and legs) of the left side of the body are described and measured.

Terminology used for parts of copulatory organs in the text and figures follows Wang (2002) with some modifications. Abbreviations used in the text and figures are: $\mathrm{ALE}=$ anterior lateral eye; $\mathrm{AME}=$ anterior median eye; $\mathrm{AME}-\mathrm{ALE}=$ distance between AME and ALE; AME-AME = distance between AME and AME; ALE-PLE $=$ distance between ALE and PLE; $\mathrm{d}=$ dorsal; $\mathrm{Fe}=$ femur; $\mathrm{Mt}=$ metatarsus; $\mathrm{p}=$ prolat eral $\mathrm{Pa}=$ patella; $\mathrm{PLE}=$ posterior lateral eye; $\mathrm{PME}=$ posterior median eye; $\mathrm{PME}-\mathrm{PLE}$ = distance between PME and PLE; PME-PME = distance between PME and PME; $r=$ retrolateral; $\mathrm{Ta}=$ tarsus; $\mathrm{Ti}=$ tibia; $\mathrm{v}=$ ventral. References to figures in the cited papers are listed in lowercase (fig. or figs); figures from the present paper are noted with an initial capital (Fig. or Figs).

DNA barcodes were obtained for future use: a partial fragment of the mitochondrial gene cytochrome oxidase subunit I (COI) was amplified and sequenced for two species using Primers LCO1490-oono (5'-CWACAAAYCATARRGATATTGG-3') (Folmer et al. 1994; Miller et al. 2010) and HCO2198-zz (5'-TAAACTTCCAGGTGACCAAAAAATCA-3') (Folmer et al. 1994; Zhao and Li 2016). For additional information on extraction, amplification, and sequencing procedures, see Zhao et al. (2013). All sequences were blasted in GenBank; accession numbers are provided in Table 1.

All specimens (including molecular vouchers) are deposited in the Institute of Zoology, Chinese Academy of Sciences in Beijing (IZCAS).

Table I. Voucher specimen infromation.

\begin{tabular}{l|c|c|c}
\hline \multicolumn{1}{c|}{ Species } & GenBank accession number & Sequence length & Collection localities \\
\hline L. geei sp. $\mathrm{n}$. & MF347606 & 543bp & China: Jiangxi: Wuyuan \\
\hline L. kulianganus & MF347607 & $630 \mathrm{bp}$ & China: Fujian: Fuzhou: Guling \\
\hline
\end{tabular}




\section{Taxonomy}

\section{Genus Longicoelotes Wang, 2002}

Longicoelotes Wang, 2002: 109. Type species Longicoelotes karschi Wang, 2002 from Jiangsu Province, China.

Composition. Four species of Longicoelotes in total: they are L. geei sp. n. (ठ̊), L.

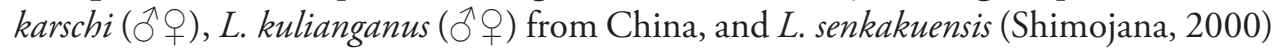
() from Ryukyu Islands.

Note. Longicolotes karschi is very similar to L. kulianganus and could be its junior synonym.

\section{Longicoelotes geei, sp. n.}

http://zoobank.org/AD46BF47-3AB3-42AD-97D9-7E4E39D83D28

Figs 1-2, 5-6

Type material. Holotype $\delta$ : China: Jiangxi Province: Wuyuan County, Lianhua Cave, N2929'02", E117³6'53", 350 m, 4.XII.2016, X. Zhang; Paratypes: 3 ㅇ 2 ${ }^{\circ}$, same

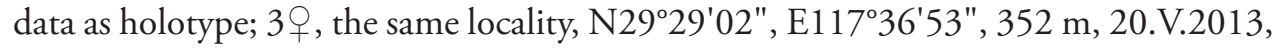
Y. Luo and J. Liu.

Etymology. The species is named after Mr. Nathaniel Gist Gee. Mr. Gee was an American biologist who lived in China about 35 years. He contributed greatly to the development of biological research and education in China, including the establishment of the first biology department in the history of university education in China (Fu 2016). He collected the holotype of L. kulianganus.

Diagnosis. The male can be distinguished from all other Longicoelotes species by its long cymbial furrow, about $1 / 3$ length of cymbium ( $v s 1 / 4$ or $1 / 5$ in other species) (Figs 1, 3; Wang 2002: figs 312-314). The female differ from all other Longicoelotes species by the nearly heart-shaped atrium and large anterior parts of copulatory ducts, subequal to receptacles ( $v$ nearly triangular atrium and small anterior parts in $L$. kulianganus, about $1 / 2$ diameter of receptacles; nearly rounded atrium and extremely small anterior parts in L. senkakuensis, about 1/4 diameter of receptacles) (Figs 2, 4-5; Shimojana 2000: figs 46-49).

Description. Male (holotype): Total length 6.00. Carapace 2.50 long, 2.00 wide. Abdomen 3.50 long, 1.75 wide. Eye sizes and interdistances: AME 0.15, ALE 0.13, PME 0.15, PLE 0.10; AME-AME 0.05, AME-ALE 0.04, PME-PME 0.05, PME-PLE 0.06. Leg measurements: I: 12.45 (3.30, 4.00, 3.00, 2.15); II: 11.30 (3.25, 3.30, 2.75, 2.00); III: 10.65 (3.00, 3.25, 2.75, 1.65); IV: 14.65 (4.00, 4.25, $4.15,2.25)$. Carapace greenish, with black lateral margins, the radial grooves distinct. Abdomen yellow, with blackish herringbone patterns. Palp as in Fig. 1: patellar apophysis long, about 2 times longer than tibia; tibia short, about $1 / 5$ length of cym- 


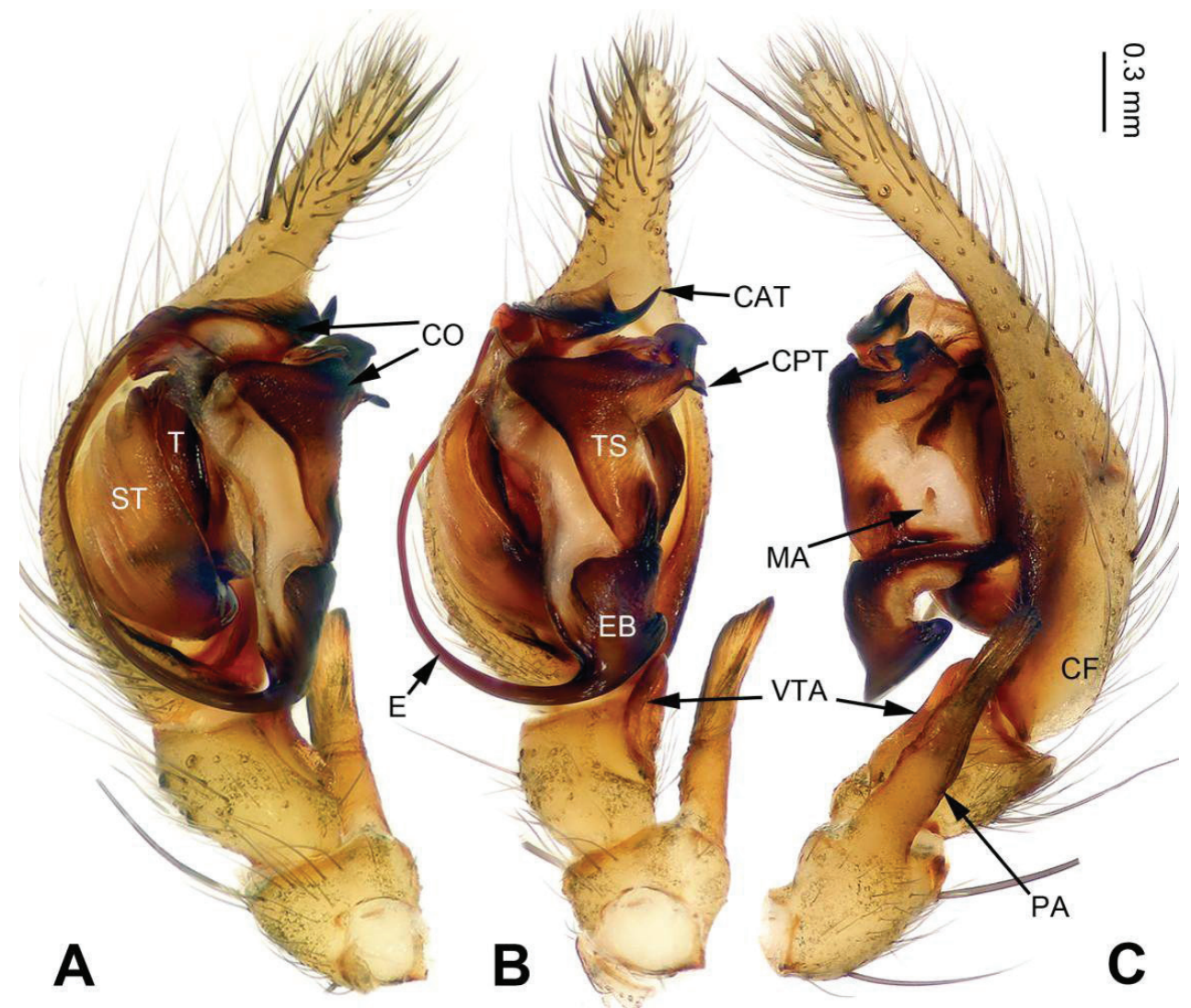

Figure I. Palp of Longicoelotes geei sp. n., holotype male. A Prolateral view B Ventral view C Retrolateral view. $\mathrm{CAT}=$ anterior tip of conductor, $\mathrm{CF}=$ cymbial furrow, $\mathrm{CO}=$ conductor, $\mathrm{CPT}=$ posterior tip of conductor, $\mathrm{E}=$ embolus, $\mathrm{EB}=$ embolic base, $\mathrm{MA}=$ median apophysis, $\mathrm{PA}=$ patellar apophysis, $\mathrm{ST}$ = subtegulum, $\mathrm{T}=$ tegulum, $\mathrm{TS}=$ tegular sclerite, $\mathrm{VTA}=$ ventral tibial apophysis. Scale bar: equal for $\mathbf{A}, \mathbf{B}, \mathbf{C}$.

bium; cymbial furrow long, about 1/3 length of cymbium; ventral tibial apophysis as long as tibia, without pointed tip, extending beyond the tibia; conductor slender and bifurcated; posterior tip of conductor bifurcated; anterior tip of conductor bifurcated, ventral part sharp, dorsal part lamellate; median apophysis reduced; embolus beginning at 5:30 o'clock position.

Leg spination in male:

\begin{tabular}{c|c|c|c|c|c}
\hline & Fe & Pa & Ti & Mt & Ta \\
\hline I & $3 \mathrm{~d} 2 \mathrm{p} 1 \mathrm{r}$ & - & $1 \mathrm{p} \mathrm{3-3v}$ & $2 \mathrm{p} 3-3 \mathrm{v}$ & - \\
\hline II & $3 \mathrm{~d} 3 \mathrm{p} 2 \mathrm{r}$ & $1 \mathrm{~d} 1 \mathrm{p}$ & $2 \mathrm{p} 3-3 \mathrm{v}$ & $1 \mathrm{p} 3-3 \mathrm{v}$ & - \\
\hline III & $3 \mathrm{~d} 2 \mathrm{p} 2 \mathrm{r}$ & $2 \mathrm{~d} 1 \mathrm{p} 1 \mathrm{r}$ & $2 \mathrm{~d} 2 \mathrm{p} 3 \mathrm{r} 3-3 \mathrm{v}$ & $2 \mathrm{~d} 5 \mathrm{p} 5 \mathrm{r} 3-3 \mathrm{v}$ & $1 \mathrm{p} \mathrm{r}$ \\
\hline IV & $3 \mathrm{~d} 2 \mathrm{p} 1 \mathrm{r}$ & $2 \mathrm{~d} 1 \mathrm{p} 1 \mathrm{r}$ & $2 \mathrm{~d} 2 \mathrm{p} 2 \mathrm{r} 3-3 \mathrm{v}$ & $5 \mathrm{p} 5 \mathrm{r} 3-3 \mathrm{v}$ & $2 \mathrm{p} 1 \mathrm{r}$ \\
\hline
\end{tabular}




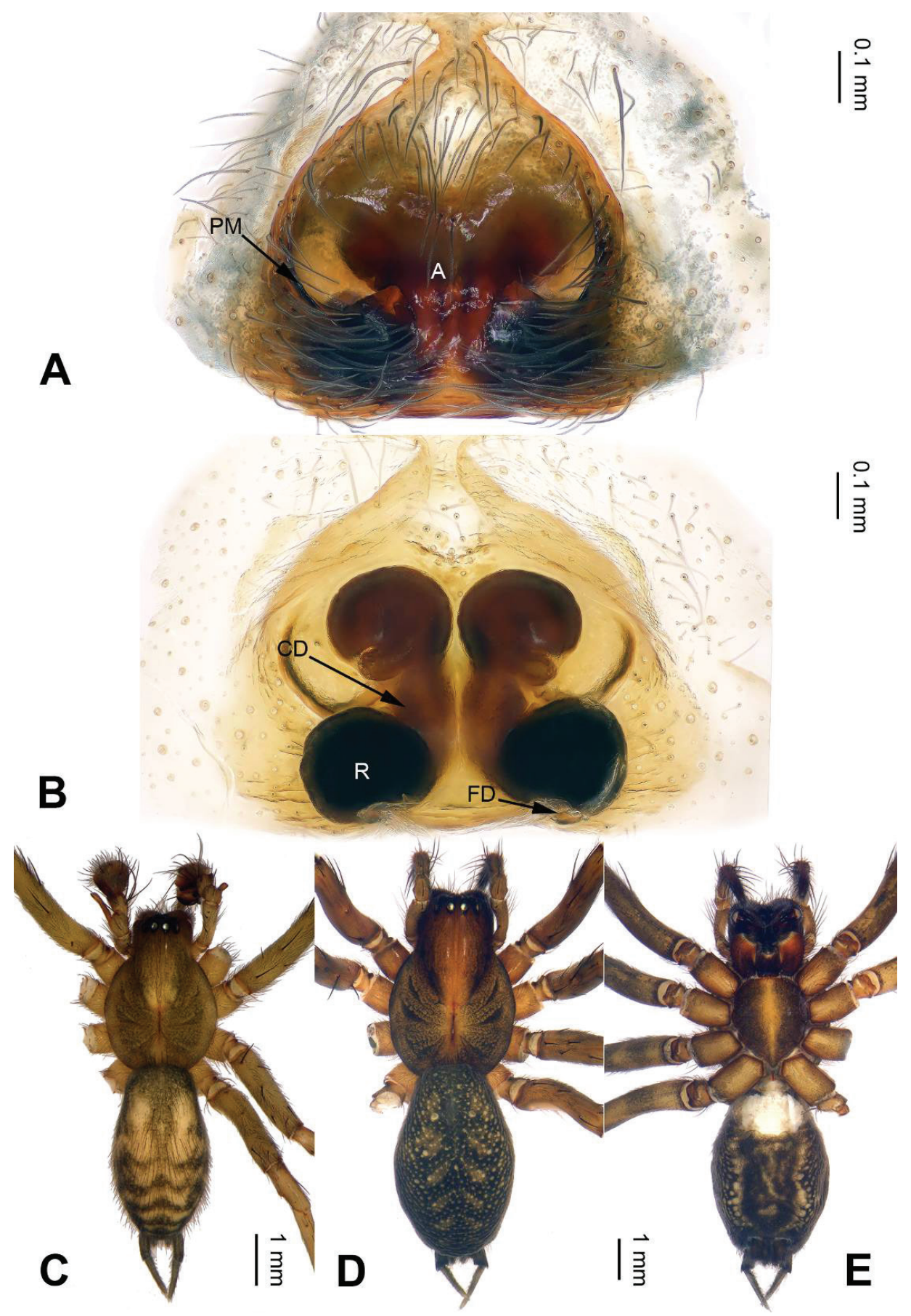

Figure 2. Longicoelotes geei sp. n., female paratype and male holotype. A Epigyne, ventral view B Vulva, dorsal view $\mathbf{C}$ Male habitus, dorsal view $\mathbf{D}$ Female habitus, dorsal view $\mathbf{E}$ Female habitus, ventral view. $\mathrm{A}=$ epigynal atrium, $\mathrm{CD}=$ copulatory duct, $\mathrm{FD}=$ fertilization duct, $\mathrm{PM}=$ posterior margin of atrium, $\mathrm{R}=$ receptacle. Scale bars: equal for $\mathbf{D}, \mathbf{E}$. 
Female (paratype): Total length 8.65. Carapace 4.10 long, 3.00 wide. Abdomen 4.55 long, 2.55 wide. Eye sizes and interdistances: AME 0.20, ALE 0.18, PME 0.18, PLE 0.15; AME-AME 0.08, AME-ALE 0.05, PME-PME 0.10, PME-PLE 0.10. Leg measurements: I: 14.25 (4.00, 5.00, 3.25, 2.00); II: 12.20 (3.50, 4.00, 3.00, 1.70); III: 11.35 (3.25, 3.75, 2.75, 1.60); IV: 15.95 (4.50, 5.10, 4.25, 2.10). Carapace reddish, with black lateral margins, the radial grooves distinct; sternum with light stripes. Abdomen black, with yellow spots and herringbone patterns. Epigyne as in Fig. 2: atrium nearly heart-shaped, length subequal to width; copulatory openings indistinct; copulatory ducts long, about 1.5 times longer than diameter of receptacles, touching each other; receptacles separated, about 1/2 diameter of receptacles; hoods indistinct.

Leg spination in female:

\begin{tabular}{c|c|c|c|c|c}
\hline & Fe & Pa & Ti & Mt & Ta \\
\hline I & $3 \mathrm{~d} 2 \mathrm{p} 1 \mathrm{r}$ & - & $1 \mathrm{p} 3-3 \mathrm{v}$ & $1 \mathrm{p} 3-3 \mathrm{v}$ & - \\
\hline II & $3 \mathrm{~d} 2 \mathrm{p} \mathrm{2r}$ & - & $2 \mathrm{p} 3-3 \mathrm{v}$ & $3 \mathrm{p} 1 \mathrm{r} 3-3 \mathrm{v}$ & - \\
\hline III & $3 \mathrm{~d} 2 \mathrm{p} 2 \mathrm{r}$ & $1 \mathrm{~d} 1 \mathrm{p}$ & $2 \mathrm{~d} 2 \mathrm{p} 2 \mathrm{r} 3-3 \mathrm{v}$ & $5 \mathrm{p} 5 \mathrm{r} 3-3 \mathrm{v}$ & $1 \mathrm{r}$ \\
\hline IV & $3 \mathrm{~d} 1 \mathrm{p} 1 \mathrm{r}$ & $1 \mathrm{~d} 1 \mathrm{r}$ & $2 \mathrm{~d} 2 \mathrm{p} 2 \mathrm{r} 3-3 \mathrm{v}$ & $1 \mathrm{~d} 5 \mathrm{p} 5 \mathrm{r} 3-3 \mathrm{v}$ & $1 \mathrm{p} 1 \mathrm{r}$ \\
\hline
\end{tabular}

Distribution. Known only from Jiangxi Province of China (Fig. 6).

\section{Longicoelotes kulianganus (Chamberlin, 1924)}

Figs 3-6

Coelotes kulianganus Chamberlin, 1924: 24, pl. 5, fig. 40 (ㅇ).

Longicoelotes kulianganus: Wang 2003: 560 (transferred from Coelotes).

Material examined. $2 \uparrow 3 \widehat{\jmath}$, China: Fujian Province: Fuzhou Prefecture: Guling (new name for Kuliang), Liushanwang Park, N2605'34", E1 19²3'32", 725 m, 1.XII.2016, $\mathrm{X}$. Zhang; $2980^{\lambda}$, the same locality, Yixia Villa, N2605'32", E119²3'32", $718 \mathrm{~m}$,

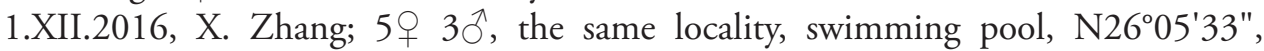
E119²3'30", 684 m, 2.XII.2016, X. Zhang.

Diagnosis. The male can be distinguished from other Longicoelotes species by indistinct median apophysis and short cymbial furrow, about $1 / 5$ length of cymbium (vs 1/3 in L. geei sp. n.) (Figs 1, 3; Wang 2002: figs 312-314). The female can be separated from other congeners by its nearly triangular atrium and rounded anterior parts of copulatory ducts ( $v s$ nearly rounded atrium and extremely small anterior parts of copulatory ducts in L. senkakuensis) (Figs 4-5; Shimojana 2000: figs 46-49).

Description. Male: Total length 8.00. Carapace 3.50 long, 2.75 wide. Abdomen 4.50 long, 2.50 wide. Eye sizes and interdistances: AME 0.20, ALE 0.18, PME 0.19, PLE 0.15; AME-AME 0.05, AME-ALE 0.05, PME-PME 0.08, PME-PLE 0.08. Leg measurements: I: 14.75 (4.25, 4.75, 3.50, 2.25); II: 12.35 (3.50, 4.00, 3.00, 1.85); III: $11.80(3.30,3.50,3.25,1.75)$; IV: 16.25 (4.50, 5.00, 4.50, 2.25). Carapace greenish, 


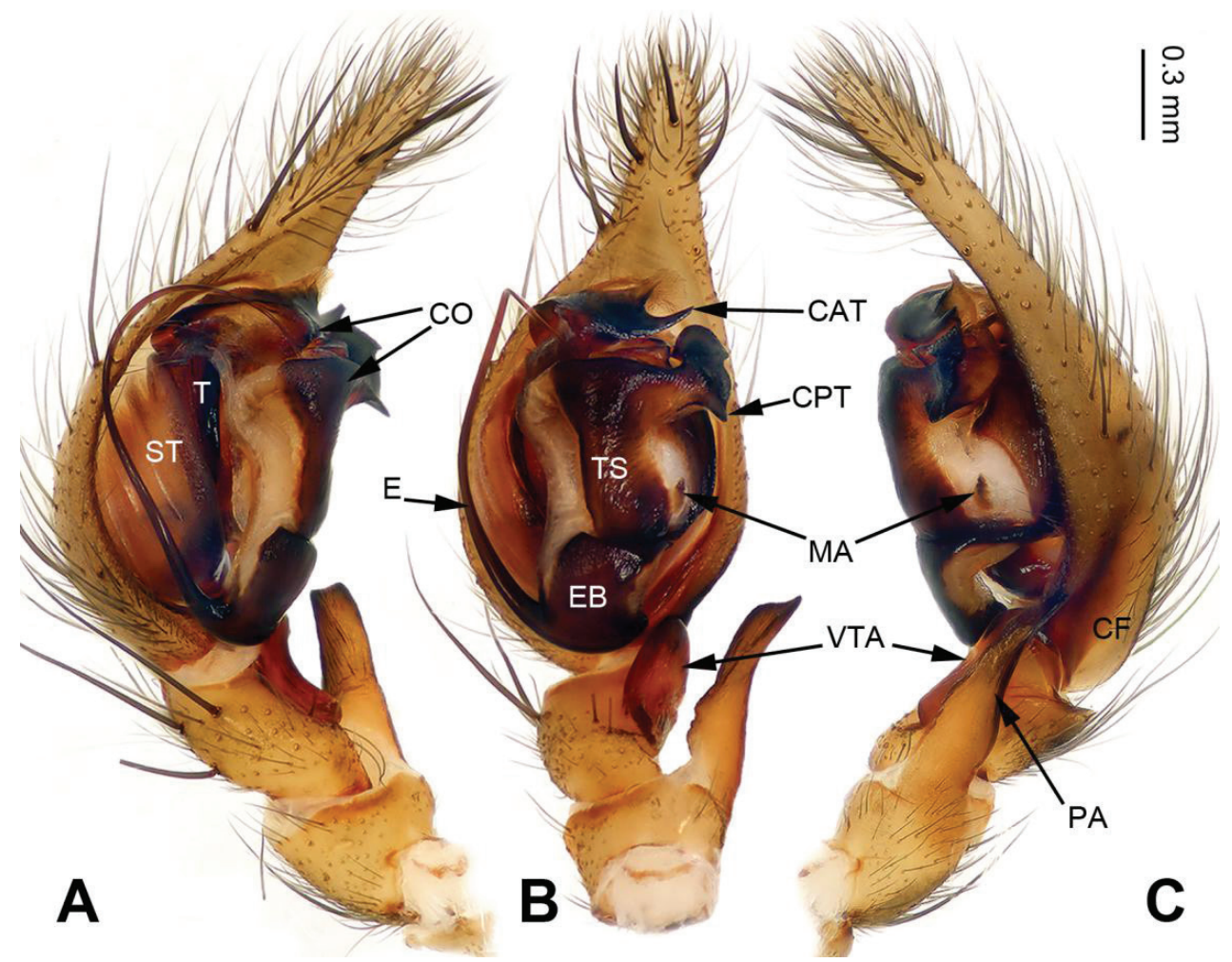

Figure 3. Palp of Longicoelotes kulianganus, specimen from Guling. A Prolateral view B Ventral view C Retrolateral view. $\mathrm{CAT}=$ anterior tip of conductor, $\mathrm{CF}=$ cymbial furrow, $\mathrm{CO}=$ conductor, $\mathrm{CPT}=$ posterior tip of conductor, $\mathrm{E}=$ embolus, $\mathrm{EB}=$ embolic base, $\mathrm{MA}=$ median apophysis, $\mathrm{PA}=$ patellar apophysis, $\mathrm{ST}=$ subtegulum, $\mathrm{T}=$ tegulum, $\mathrm{TS}=$ tegular sclerite, $\mathrm{VTA}=$ ventral tibial apophysis. Scale bar: equal for $\mathbf{A}, \mathbf{B}, \mathbf{C}$.

the radial grooves indistinct. Abdomen grayish, with blackish herringbone patterns. Palp as in Fig. 3: patellar apophysis long, about 2 times longer than tibia; tibia short, about $1 / 4$ length of cymbium; cymbial furrow short, about 1/5 length of cymbium; ventral tibial apophysis subequal to the tibial length, without pointed tip; conductor broad and bifurcated; posterior tip of conductor bifurcated; anterior tip of conductor bifurcated, ventral part nearly spine-shaped, dorsal part lamellate; median apophysis indistinct; embolus beginning at 6:00 o'clock position.

Leg spination in male:

\begin{tabular}{c|c|c|c|c|c}
\hline & Fe & Pa & Ti & Mt & Ta \\
\hline I & $3 \mathrm{~d} 2 \mathrm{p} 1 \mathrm{r}$ & - & $3-3 \mathrm{v}$ & $1 \mathrm{p} 3-3 \mathrm{v}$ & - \\
\hline II & $3 \mathrm{~d} 1 \mathrm{p} 1 \mathrm{r}$ & - & $2 \mathrm{p} 3-3 \mathrm{v}$ & $3 \mathrm{p} 3-3 \mathrm{v}$ & - \\
\hline III & $3 \mathrm{~d} 2 \mathrm{p} \mathrm{2r}$ & $1 \mathrm{~d} 1 \mathrm{p} 1 \mathrm{r}$ & $2 \mathrm{~d} 2 \mathrm{p} 2 \mathrm{r} 3-3 \mathrm{v}$ & $5 \mathrm{p} 5 \mathrm{r} 3-3 \mathrm{v}$ & $2 \mathrm{p} 1 \mathrm{r}$ \\
\hline IV & $3 \mathrm{~d} 2 \mathrm{p} 1 \mathrm{r}$ & $1 \mathrm{~d} 1 \mathrm{p} 1 \mathrm{r}$ & $2 \mathrm{~d} 2 \mathrm{p} 2 \mathrm{r} 3-3 \mathrm{v}$ & $5 \mathrm{p} 5 \mathrm{r} 3-3 \mathrm{v}$ & $2 \mathrm{p} 1 \mathrm{r}$ \\
\hline
\end{tabular}




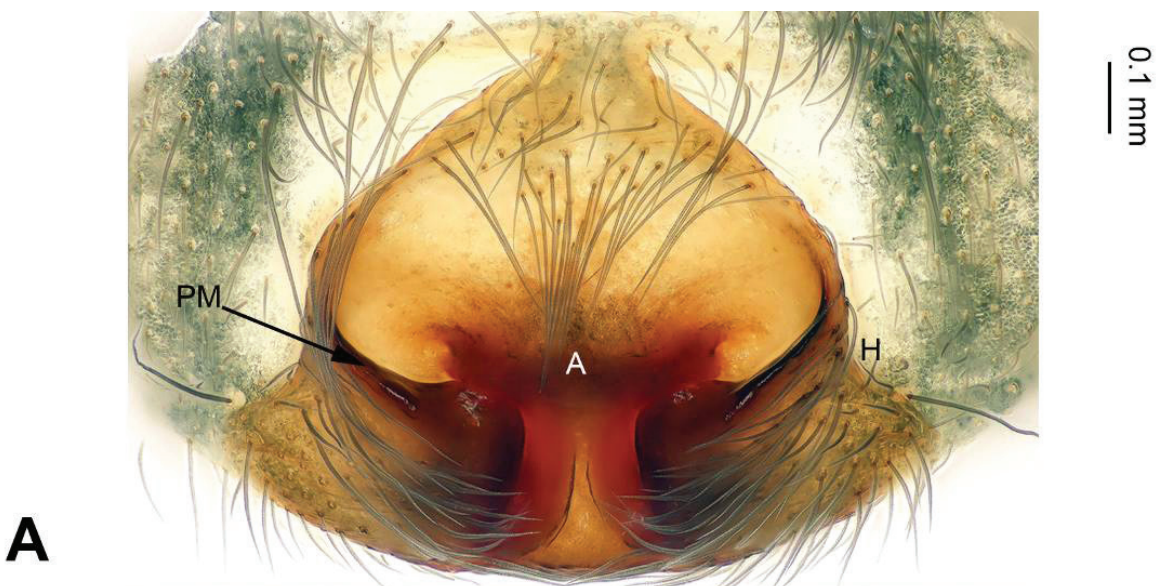

\section{$\stackrel{\circ}{3}$}

$\mid \stackrel{0}{3}$
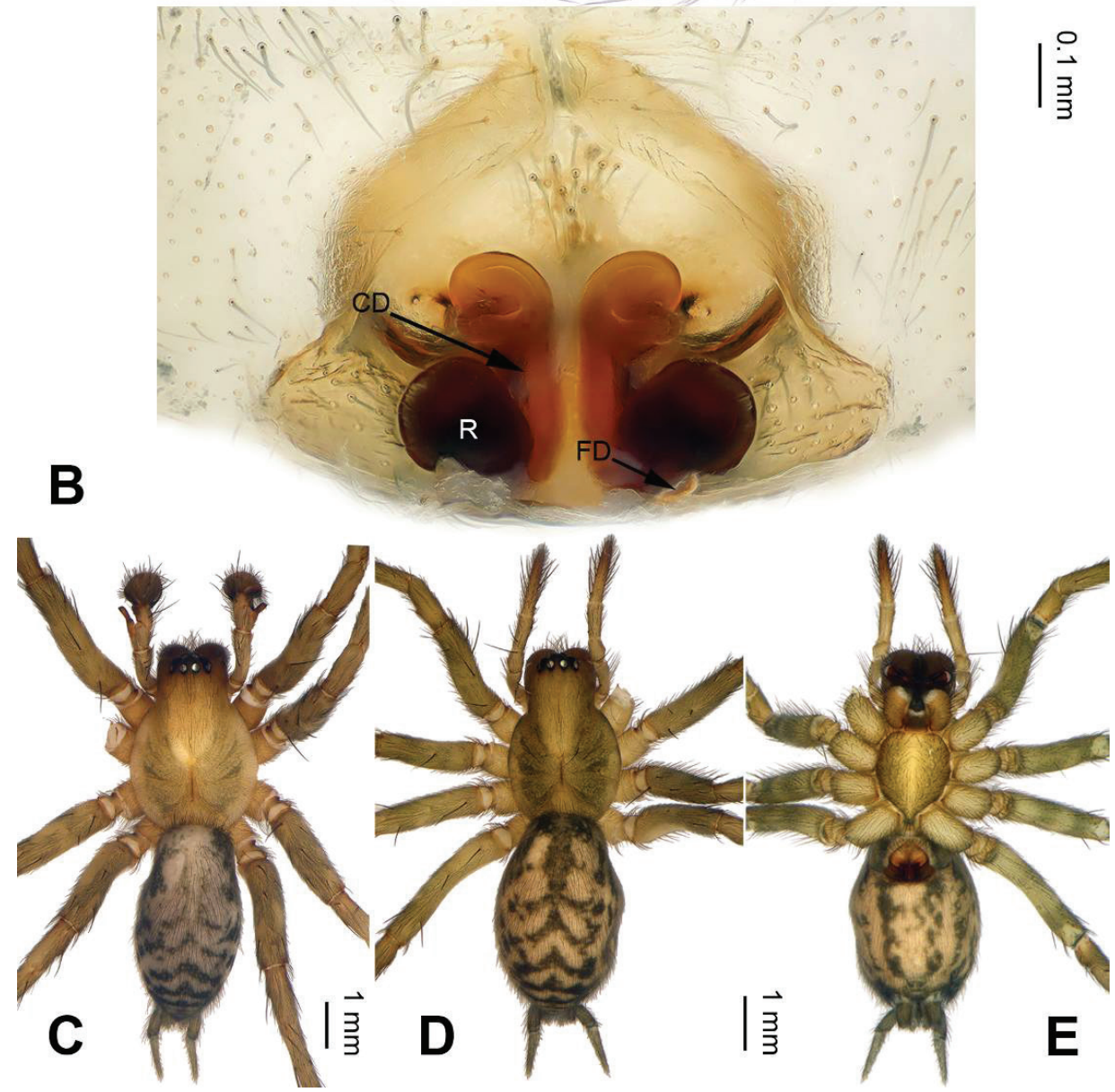

Figure 4. Longicoelotes kulianganus, specimen from Guling. A Epigyne, ventral view B Vulva, dorsal view C Male habitus, dorsal view D Female habitus, dorsal view $\mathbf{E}$ Female habitus, ventral view. $A=$ epigynal atrium, $\mathrm{CD}=$ copulatory duct, $\mathrm{FD}=$ fertilization duct, $\mathrm{H}=$ epigynal hood, $\mathrm{PM}=$ posterior margin of atrium, $\mathrm{R}$ = receptacle. Scale bars: equal for $\mathbf{D}, \mathbf{E}$. 

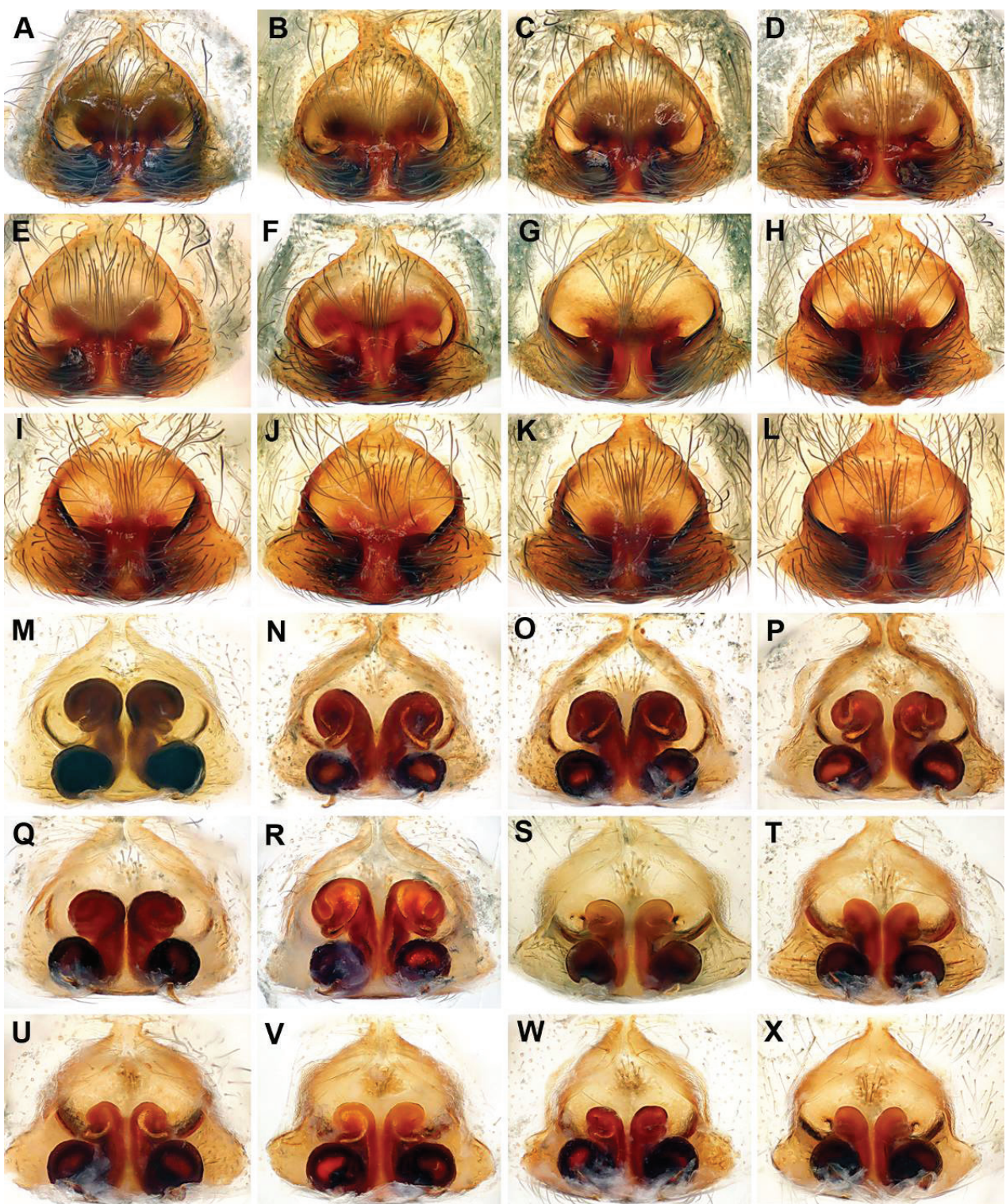

Figure 5. Longicoelotes geei sp. n. (A-F, M-R) and L. kulianganus (G-L, S-X), variations in shape of epigynal atrium and size of copulatory ducts. A-L Epigyne, ventral view $\mathbf{M}-\mathbf{X}$ Vulva, dorsal view.

Female: Total length 6.75. Carapace 2.75 long, 2.15 wide. Abdomen 4.00 long, 2.25 wide. Eye sizes and interdistances: AME 0.15, ALE 0.15, PME 0.16, PLE 0.13; AME-AME 0.06, AME-ALE 0.05, PME-PME 0.10, PME-PLE 0.10. Leg measurements: I: 10.50 (3.00, 3.50, 2.50, 1.50); II: 9.20 (2.75, 3.00, 2.25, 1.20); III: 8.70 $(2.50,2.70,2.25,1.25)$; IV: 12.00 (3.50, 3.75, 3.25, 1.50). Carapace greenish, with black lateral margins, the radial grooves distinct; sternum with light stripes. Abdo- 


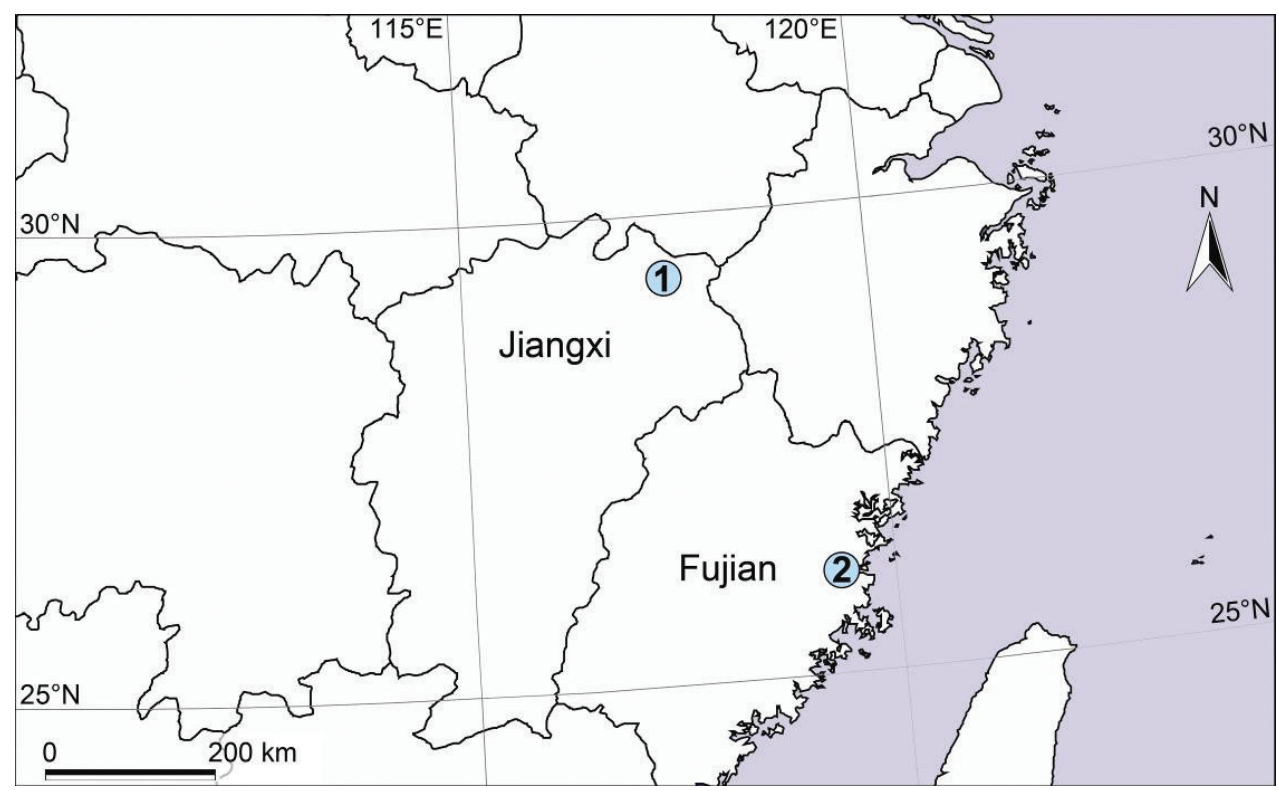

Figure 6. Collecting localities of two Longicoelotes species from China. I. L. geei sp. n., 2. L. kulianganus.

men black, with yellow spots and herringbone patterns. Epigyne as in Fig. 4: atrium with well delimited posterior margin, length subequal to width; copulatory openings indistinct, hidden by posterior margin of atrium; copulatory ducts long, about 2 times longer than diameter of receptacles; receptacles widely separated, subequal to diameter receptacles; hoods distinct.

Leg spination in female:

\begin{tabular}{c|c|c|c|c|c}
\hline & Fe & Pa & Ti & Mt & Ta \\
\hline I & $3 \mathrm{~d} 2 \mathrm{p} 1 \mathrm{r}$ & - & $1 \mathrm{p} 3-3 \mathrm{v}$ & $3-3 \mathrm{v}$ & - \\
\hline II & $3 \mathrm{~d} 2 \mathrm{p} 2 \mathrm{r}$ & $2 \mathrm{~d} 1 \mathrm{p}$ & $2 \mathrm{p} 3-3 \mathrm{v}$ & $3 \mathrm{p} 1 \mathrm{r} 3-3 \mathrm{v}$ & - \\
\hline III & $3 \mathrm{~d} 2 \mathrm{p} 2 \mathrm{r}$ & $2 \mathrm{~d} 1 \mathrm{p} 1 \mathrm{r}$ & $2 \mathrm{p} 3 \mathrm{r} 3-3 \mathrm{v}$ & $5 \mathrm{p} 5 \mathrm{r} 3-3 \mathrm{v}$ & $1 \mathrm{p} 1 \mathrm{r}$ \\
\hline IV & $3 \mathrm{~d} 1 \mathrm{p} 1 \mathrm{r}$ & $1 \mathrm{~d} 1 \mathrm{p} 1 \mathrm{r}$ & $2 \mathrm{~d} 2 \mathrm{p} 2 \mathrm{r} 3-3 \mathrm{v}$ & $5 \mathrm{p} 5 \mathrm{r} 3-3 \mathrm{v}$ & $1 \mathrm{p} 1 \mathrm{r}$ \\
\hline
\end{tabular}

Distribution. Known only from Fujian Province of China (Fig. 6).

\section{Acknowledgements}

The manuscript benefitted greatly from comments by Yuri M. Marusik (Magadan, Russia) and Alexander A. Fomichev (Barnaul, Russia). Joseph K. H. Koh (Singapore) checked the language. This study was supported by the National Natural Sciences Foundation of China $(31471960,31530067)$ and the Southeast Asia Biodiversity Research Institute, Chinese Academy of Sciences (2015CASEABRI005, Y4ZK111B01). 


\section{References}

Chamberlin RV (1924) Descriptions of new American and Chinese spiders, with notes on other Chinese species. Proceedings of the United States National Museum 63(13): 1-38. https://doi.org/10.5479/si.00963801.63-2481.1

Fu L (2016) Nathaniel Gist Gee's contribution to biology in modern China. Protein \& Cell 8(4): 237-239. https://doi.org/10.1007/s13238-016-0318-x

Folmer O, Black M, Hoeh W, Lutz R, Vrijenhoek R (1994) DNA primers for amplification of mitochondrial cytochrome $c$ oxidase subunit I from diverse metazoan invertebrates. Molecular Marine Biology and Biotechnology 3(5): 294-299.

Miller JA, Carmichael A, Ramirez MJ, Spagna JC, Haddad CR, Řezáč M, Johannesen J, Král J, Wang XP, Griswold CE (2010) Phylogeny of entelegyne spiders: affinities of the family Penestomidae (new rank), generic phylogeny of Eresidae, and asymmetric rates of change in spinning organ evolution (Araneae, Araneoidea, Entelegynae). Molecular Phylogenetics and Evolution 55: 786-804. https://doi.org/10.1016/j.ympev.2010.02.021

Shimojana M (2000) Description of eleven new species of the genus Coelotes (Araneae: Amaurobiidae) from the Ryukyu Islands, Japan. Acta Arachnologica 49: 165-189. https://doi. org/10.2476/asjaa.49.165

Wang XP (2002) A generic-level revision of the spider subfamily Coelotinae (Araneae, Amaurobiidae). Bulletin of the American Museum of Natural History 269: 1-150. https://doi. org/10.1206/0003-0090(2002)269<0001:AGLROT>2.0.CO;2

Wang XP (2003) Species revision of the coelotine spider genera Bifidocoelotes, Coronilla, Draconarius, Femoracoelotes, Leptocoelotes, Longicoelotes, Platocoelotes, Spiricoelotes, Tegecoelotes, and Tonsilla (Araneae: Amaurobiidae). Proceedings of the California Academy of Sciences 54: 499-662.

World Spider Catalog (2017) World Spider Catalog. Natural History Museum Bern. http:// wsc.nmbe.ch [version 18.0, accessed on June 5, 2017]

Zhao Z, Li S (2016) Papiliocoelotes gen. n., a new genus of Coelotinae (Araneae, Agelenidae) spiders from the Wuling Mountains, China. ZooKeys 585: 33-50. https://doi.org/10.3897/ zookeys. 585.8007

Zhao Z, Su TJ, Chesters D, Wang SD, Ho SYW, Zhu CD, Chen XL, Zhang CT (2013) The mitochondrial genome of Elodia flavipalpis Aldrich (Diptera: Tachinidae) and the evolutionary timescale of tachinid flies. PLoS ONE 8:e61814. https://doi.org/10.1371/journal. pone. 0061814 